EPJ Web of Conferences 63,01015 (2013)

DOI: $10.1051 /$ epjconf/ 20136301015

C Owned by the authors, published by EDP Sciences, 2013

\title{
Study of $0^{+}$States at iThemba LABS
}

P. Jones ${ }^{1, a}$, P. Papka ${ }^{1,2}$, J.F. Sharpey-Shafer ${ }^{3}$, P. Vymers ${ }^{2}$, S. P. Bvumbi ${ }^{4}$, T. D. Bucher ${ }^{1}$, T. S. Dinoko ${ }^{3}$, J. L. Easton ${ }^{1,3}$, M. S. Herbert $^{3}$, B. V. Kheswa ${ }^{1,2}$, N. Khumalo ${ }^{5}$, E. A. Lawrie ${ }^{1}$, J. J. Lawrie ${ }^{1}$, S. N. T. Majola ${ }^{1,6}$, J. Ndayishimye ${ }^{1,2}$, D. Negi ${ }^{1}$, S. P. Noncolela $^{1,3}$, J. N. Orce ${ }^{3}$, O. Shirinda ${ }^{1}$, P. Sithole ${ }^{1,3}$, M. A. Stankiewicz ${ }^{1,6}$ and M. Wiedeking ${ }^{1}$

${ }^{1}$ iThemba Laboratory for Accelerator Based Sciences, National Research Foundation, P/B 722 Somerset West, 7129 South Africa

${ }^{2}$ Department of Physics, University of Stellenbosch, P/B X1 Matieland, 7602 South Africa

${ }^{3}$ Department of Physics, University of the Western Cape, P/B X17 Bellville, 7535 South Africa

${ }^{4}$ University of Johannesburg, Department of Physics, PO Box 524, Auckland Park, 2006 South Africa

${ }^{5}$ University of Zululand, Department of Physics, P/B X1001, Kwa Dlangezwa, 3886 South Africa

${ }^{6}$ University of Cape Town, Department of Physics, P/B X3, Rondebosch, 7701 South Africa

\begin{abstract}
An initial series of experiments utilising the $\left({ }^{3} \mathrm{He}, \mathrm{n}\right)$ reactions have been carried out at iThemba LABS. These are complimentary to transfer reactions such as $(t, p)$ stripping or $(p, t)$ pick-up reactions measurements using magnetic spectrometers. However, in the past, $\left({ }^{3} \mathrm{He}, \mathrm{n}\right)$ measurements have suffered from ambiguities due to the low energy resolution inbuilt into the time-of-flight techniques used. By combining neutron detection techniques with the AFRODITE $\gamma$-ray array, it has been shown that very good energy resolutions can be achieved. This enables the relative strengths of two proton stripping to excited states, separated by only a few $\mathrm{keV}$, to be accurately measured. This technique has been applied to first excited $0_{2}+$ states and, in particular to those uniquely seen in double $\beta$-decay.
\end{abstract}

\section{Introduction}

The study of excited $0^{+}$states provides information on how nucleons are paired and their associated structures in the nucleus [1]. The first excited $0_{2}{ }^{+}$states in $\mathrm{N}=90$ nuclei have been shown not to be $\beta$-vibrations of the nuclear shape [2]. It has been demonstrated that they are $2 \mathrm{p}-2 \mathrm{~h}$ neutron paring vibrational states forming a signature zero "second vacuum" [3,4]. There are ample one and two neutron direct transfer data to these states but a paucity of two proton transfer data. Accurate two proton transfer data would give valuable information on the role of proton pairing in the microscopic structure of $0^{+}$states.

Reactions where protons are stripped off the beam nucleus, so that the outgoing particle is a neutron, are difficult to carry out. In particular it is especially challenging to obtain sufficient neutron energy resolution [5]. Examples of these neutron emitting direct reactions are $(\mathrm{d}, \mathrm{n})$ and $\left({ }^{3} \mathrm{He}, \mathrm{n}\right)$ which donate one and two protons to the target nucleus respectively. One proton stripping can be made with the $\left({ }^{3} \mathrm{He}, \mathrm{d}\right)$ reaction, but two proton stripping requires reactions using heavy ions such as $\left({ }^{16} \mathrm{O},{ }^{14} \mathrm{C}\right)$ and very good resolutions are still difficult to achieve. Furthermore, interaction with the outgoing particle introduces possible complications due to the structure of ${ }^{14} \mathrm{C}$. The $\left({ }^{3} \mathrm{He}, \mathrm{n} \gamma\right)$ reaction has been used to study the two proton content of excited $0_{\mathrm{n}}^{+}$states, achieving excellent energy resolution in the $\gamma$ channel.

In this contribution the apparatus used is presented and the application to the first excited $\mathrm{O}_{2}^{+}$states in ${ }^{100} \mathrm{Ru}$ and ${ }^{150} \mathrm{Sm}$ seen in double $\beta$-decay $[6,7]$. The importance of understanding the microscopic configurations of these $\mathrm{O}_{2}{ }^{+}$ states, and the ground $0_{1}^{+}$states of the parent and daughter nuclei, has been stressed in a recent review article [8]. The better the transition matrix elements can be calculated, the more accurately an effective neutrino mass can be extracted. There is also the ambition of using the double $\gamma$-ray decay from the $\mathrm{O}_{2}^{+}$states to give a fourfold coincidence with the two electrons to improve the sensitivity of experiments so that the level of $\approx 10^{24} \mathrm{y}$ partial half-life can be achieved. This is the estimated sensitivity required to detect $2 \beta 0 \mathrm{v}$ neutrinoless double decay to determine the Majorana/Dirac nature of neutrinos.

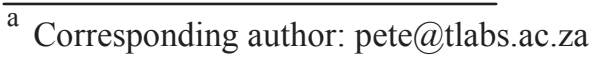




\section{Experimental Setup}

In order to establish the two paired proton components of the ${ }^{100} \mathrm{Ru}$ and ${ }^{150} \mathrm{Sm}_{2}{ }^{+}$states, a wall of neutron detectors has been constructed down-beam of the AFRODITE array to detect fast neutrons in coincidence with $\gamma$-rays from the $\left({ }^{3} \mathrm{He}, \mathrm{n}\right)$ reaction on targets of ${ }^{98} \mathrm{Mo}$ and ${ }^{148} \mathrm{Nd}$. This "direct" reaction is only $0.1 \%$ of the total crosssection. Most $\gamma$-rays come from fusion evaporation $\mathrm{xn}$ reactions whose associated neutrons have half the velocity of those from $\left({ }^{3} \mathrm{He}, \mathrm{n}\right)$.

The neutron detector setup comprises a set of 12 large volume scintillators $\left(600 \times 100 \times 100 \mathrm{~mm}^{3}\right.$ each $)$ initially built for the neutron time-of-flight setup at iThemba LABS, Cape Town, South Africa. The detectors were arranged in a $6 \times 2$ compact geometry placed at $\theta=0^{\circ}$ perpendicular to the beam axis at a distance of $2.1 \mathrm{~m}$ downstream of the target. The vertical walls of six detectors placed on the top of each other have a total thickness of $0.2 \mathrm{~m}$. A schematic of the setup is depicted in Fig.1. The beam dump is situated $50 \mathrm{~cm}$ downstream of the target.

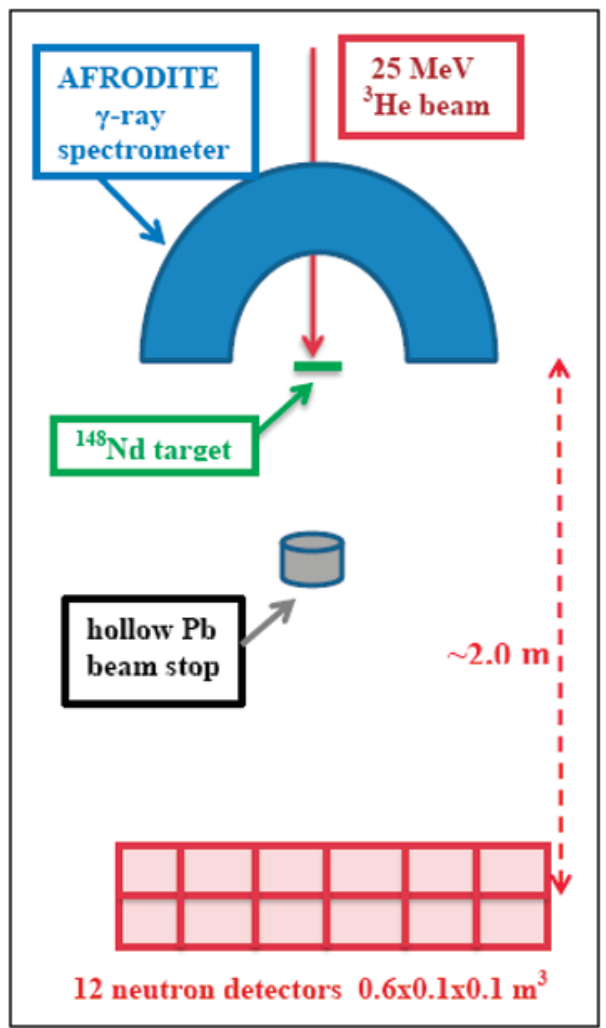

Figure 1: Schematic of experimental setup. AFRODITE has 9 HPGe "clover" detectors in BGO shields. The neutron wall has 12 detectors arranged in a double layer at zero degrees to the beam direction.

The AFRODITE detectors have been recently instrumented using digital electronics using the Pixie-16 $100 \mathrm{MHz}$ Digital Gamma Finder incorporated into the iThemba LABS Digital Data Acquisition System. The neutron scintillation detectors were also, for the first time, instrumented the same way. Here the signal from each end of the detector is used to determine the longitudinal position of the neutron interaction. Thus the timing information is of utmost important and constant-fraction timing techniques were used to determine the interaction time to 1-2 ns, relating to a position resolution of a few $\mathrm{cm}$.

\section{Experimental Method}

Initially the test reaction ${ }^{59} \mathrm{Co}\left({ }^{3} \mathrm{He}, \mathrm{n} \gamma\right){ }^{61} \mathrm{Cu}$ at a bombarding energy of $25 \mathrm{MeV}$ was employed to test proof-of-principle of the measurement apparatus. Of primary interest is the ability to discriminate between $\gamma$ rays detected in the neutron scintillation detectors from the target, beam stop and moreover statistical neutrons from $\left({ }^{3} \mathrm{He}, \mathrm{xn}\right)$ reactions, and the fast neutrons from the $\left({ }^{3} \mathrm{He}, \mathrm{n}\right)$ reactions. The latter are of interest to select the single ejected neutron channels. Typically, the ratio of the direct reaction fast neutrons to the statistical compound nucleus evaporation neutrons is 1 to $10^{3}$. A time-of-flight spectrum, measured against the cyclotron RF signal, is shown in figure 2.

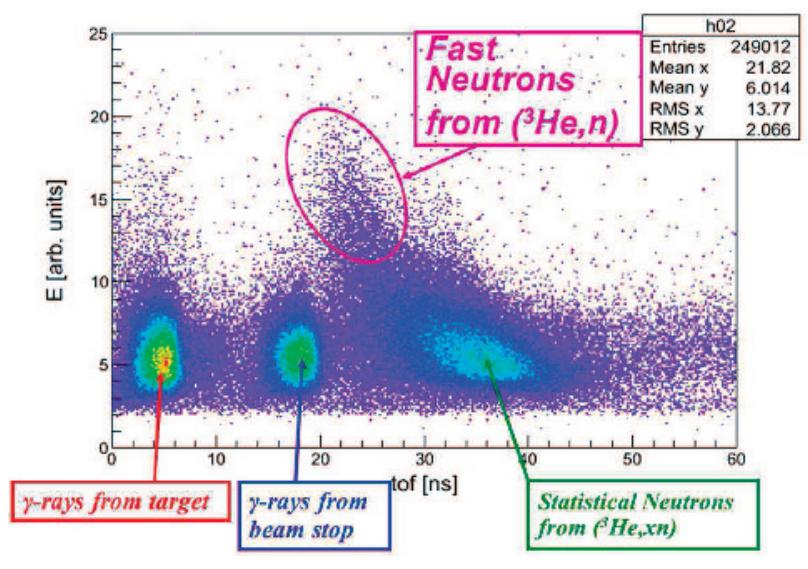

Figure 2: Time-of-flight versus energy deposited in the scintillation neutron detectors.

As the neutron detectors are positioned within $10^{\circ}$ of the beam direction, the geometry of the apparatus is designed to pick out $L=0$ two proton transfers where the emitted neutron angular distribution is peaked around a scattering angle of $0^{\circ}$. This geometry helps the selection of direct reaction neutrons as the statistical neutrons are emitted isotropically in the centre-of-mass. The final states populated will have to have the same spin as the target nucleus as no angular momentum is transferred if $L=0$.

Fig. 3 shows the total projection of all $\gamma$-rays from the ${ }^{59} \mathrm{Co}\left({ }^{3} \mathrm{He}, \mathrm{n} \gamma\right){ }^{61} \mathrm{Cu}$ reaction compared with $\gamma$-rays in coincidence with fast neutrons after a background has been subtracted. As the ground state spin of the target nucleus is $7 / 2^{-}$, one proton hole in an otherwise closed $f_{7 / 2}$ shell, the $L=0$ transfer should only populate $7 / 2^{-}$states in the residual nucleus ${ }^{61} \mathrm{Cu}$. Indeed, the decays for the three 
known $7 / 2^{-}$states in ${ }^{61} \mathrm{Cu}$ are much the strongest $\gamma$-rays in the very clean spectrum shown in Fig. 3(b).
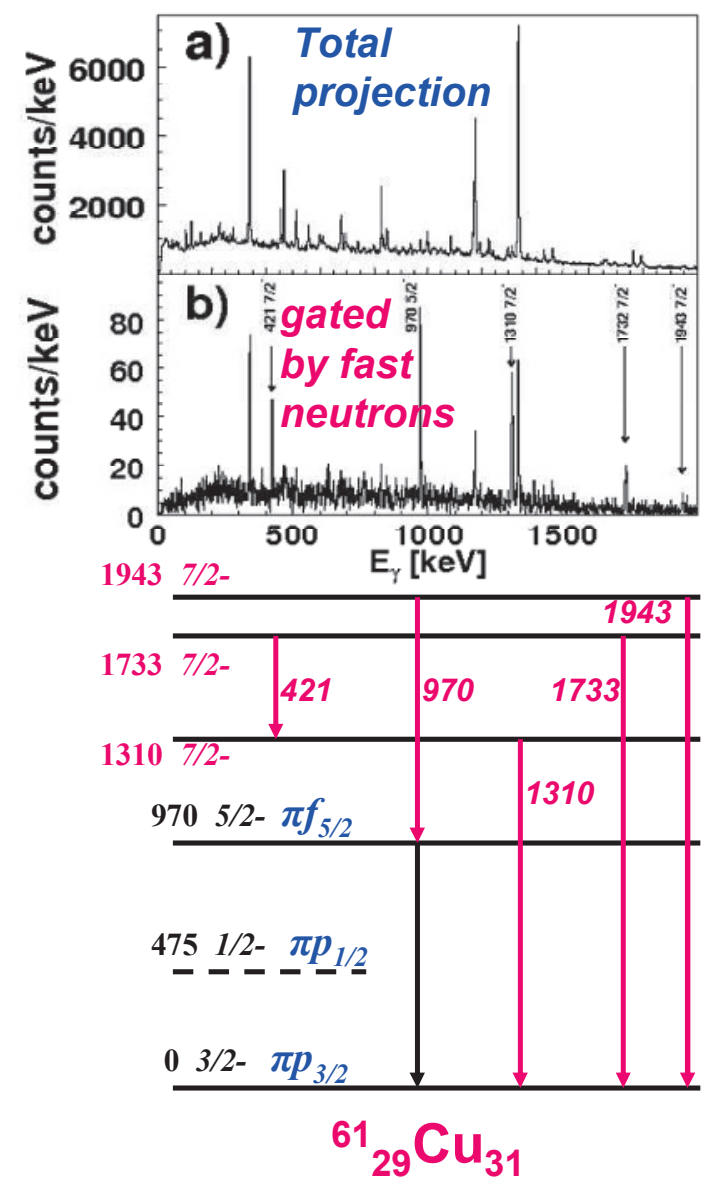

Figure 3: $\gamma$-ray spectra from the ${ }^{59} \mathrm{Co}\left({ }^{3} \mathrm{He}, \mathrm{n} \gamma\right){ }^{61} \mathrm{Cu}$ reaction; (a) the total projection of all $\gamma$-rays, (b) $\gamma$-rays in coincidence with fast neutrons gated as illustrated in figure 2 . The decay scheme underneath the spectra shows the decay of the $7 / 2^{-}$levels populated in the reaction.

It is clear that the experimental technique can be used to obtain the two proton content of closely spaced states in heavy nuclei.

\section{Preliminary Analysis}

Two experiments were performed using the $\left({ }^{3} \mathrm{He}, n\right)$ reaction on isotopically pure ${ }^{98} \mathrm{Mo}$ and ${ }^{148} \mathrm{Nd}$ targets following the technique detailed above. The preliminary spectra of Fig. 4 and 5 show that the first $\mathrm{O}_{2}{ }^{+}$excited states of ${ }^{100} \mathrm{Ru}(1130 \mathrm{keV})$ and ${ }^{150} \mathrm{Sm}(740 \mathrm{keV})$ were populated, though rather weakly for the Samarium isotope. For both nuclei, higher spin states are also populated with larger intensities. Clearly the two proton structures of the two nuclei are rather different.

A first analysis of the data shows $\gamma$-rays from the $2^{+}, 4^{+}$ and $6^{+}$ground state rotational band in ${ }^{150} \mathrm{Sm}$. However a very small $406 \mathrm{keV}$ peak is from the first excited $0_{2}{ }^{+}$ state. A problem with the experimental technique is that there is no detection of the two proton stripping to the ground state as it does not decay by $\gamma$-rays. However a way of estimating the absolute yields is to fit the relative yields of excited states to the line shapes of the low resolution time-of-flight data in Ref. [5]. Fig. 6 shows such a fit to the ground state transition seen in the ${ }^{148} \mathrm{Nd}\left({ }^{3} \mathrm{He}, \mathrm{n}\right){ }^{150} \mathrm{Sm}$ reaction at $E_{\text {lab }}=25.4 \mathrm{MeV}$ and zero degrees using a $9 \mathrm{~m}$ flight path and a time resolution of 1 ns $\sim 450 \mathrm{keV}$ (Fig. 1 of Ref. [5]). The transitions to the ground $0_{1}^{+}$state and the first excited $2_{1}^{+}$state at $334 \mathrm{keV}$ are barely resolved in the data of [5]. The fit has combined the intensities of the transitions from the $0_{2}{ }^{+}$ and $4_{1}^{+}$states decaying by $\gamma$-rays with energies of 406 and $440 \mathrm{keV}$ respectively. Table 1 compares the relative intensities of the transition strengths to the lowest states in ${ }^{150} \mathrm{Sm}$ where the data are normalised to 100 for the $2_{1}{ }^{+}$ state. The data for the $4_{1}^{+}$state are in reasonable agreement for both the $\gamma$-ray data and the time-of-flight $(t-o-f)$ data, considering the resolution of the latter and the difficulties in fitting such data. This indicates that the population of the $\mathrm{O}_{2}{ }^{+}$state is less than about $5 \%$ of the transition strength to the ground state and demonstrates that this state has only a very small component that appears as its isotone ${ }^{148} \mathrm{Nd}+$ two paired protons. This supports recent assertions $[3,4,10]$ that these first excited $\mathrm{O}_{2}^{+}$states are two neutron configurations lowered into the pairing gap by configuration dependent pairing.
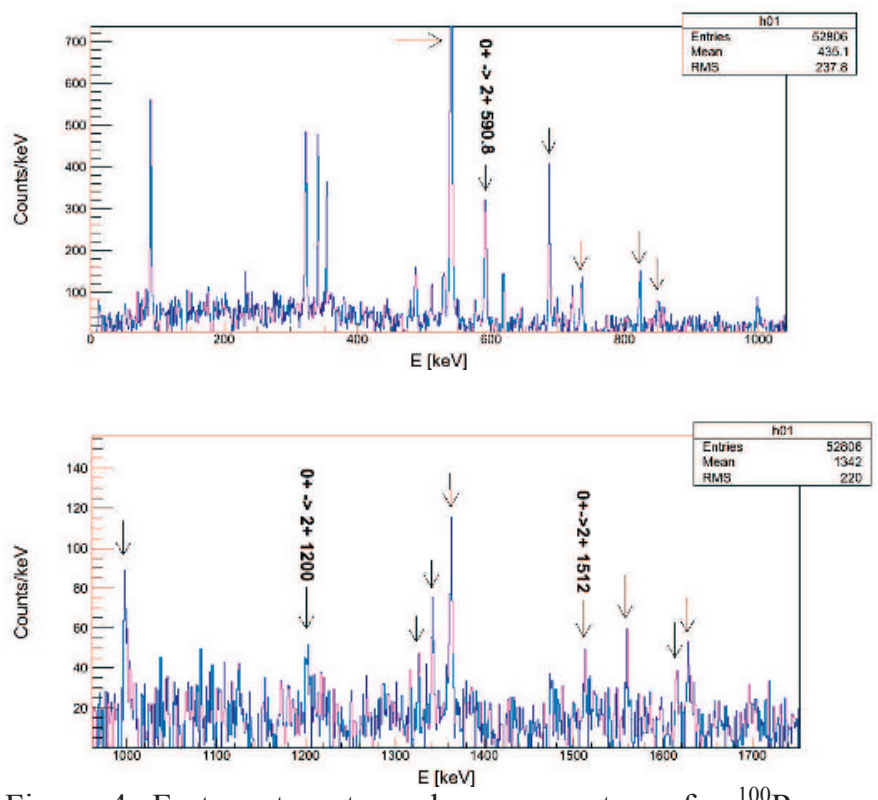

Figure 4: Fast neutron tagged $\gamma$-ray spectrum for ${ }^{100} \mathrm{Ru}$ from the $\left({ }^{3} \mathrm{He}, \mathrm{n}\right)$ reaction at a beam energy of $25 \mathrm{MeV} . \gamma-$ rays from ${ }^{100} \mathrm{Ru}$ are marked with arrows. The $591 \mathrm{keV} \gamma$ ray from the $0_{2}^{+}$state and the 1200 and $1512 \mathrm{keV} \gamma$-rays from the $\mathrm{O}_{3}{ }^{+}$and $\mathrm{O}_{4}{ }^{+}$states respectively are emphasised. 


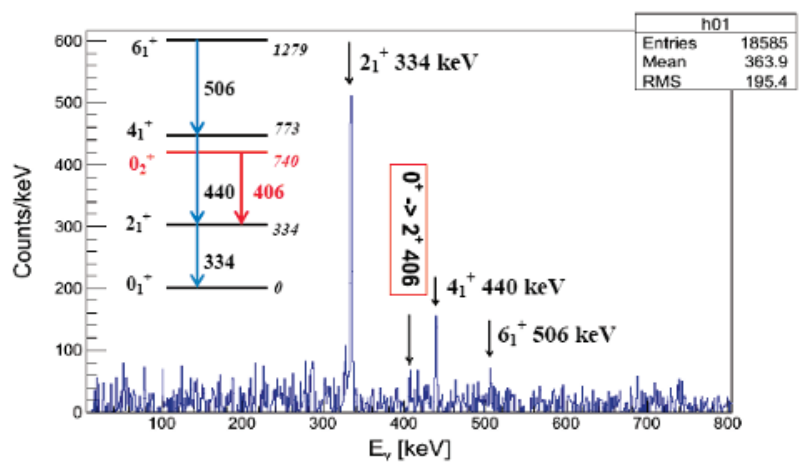

Figure 5: Fast neutron tagged gamma-ray spectrum for ${ }^{150} \mathrm{Sm}$ from the $\left({ }^{3} \mathrm{He}, \mathrm{n}\right)$ reaction at a beam energy of 25 $\mathrm{MeV}$. $\gamma$-rays from ${ }^{150} \mathrm{Sm}$ are marked with arrows. The $406 \mathrm{keV} \gamma$-ray from the $0_{2}^{+}$state is emphasised.

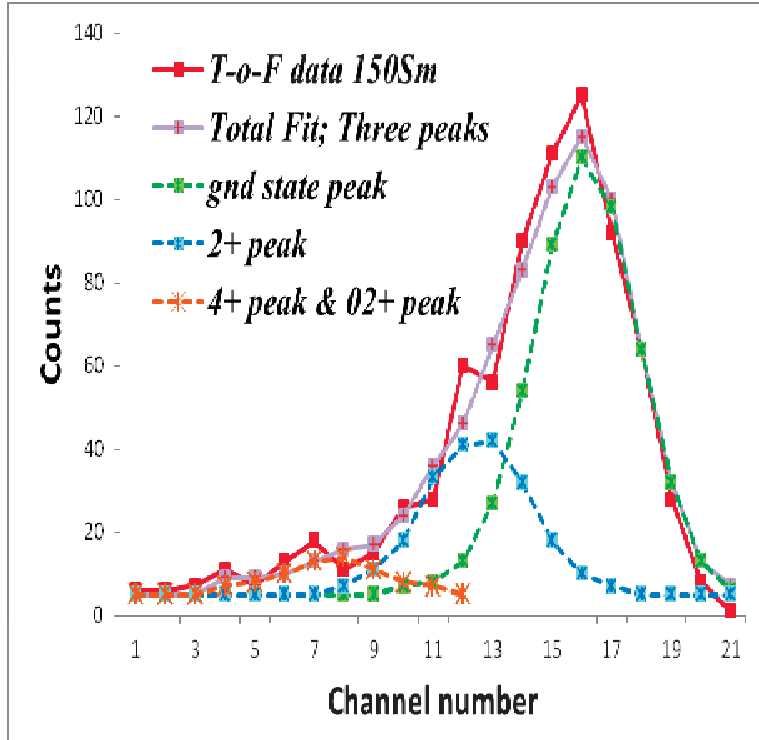

Figure 6. . Fits to the Time-of-Flight ${ }^{148} \mathrm{Nd}\left({ }^{3} \mathrm{He}, \mathrm{n}\right){ }^{150} \mathrm{Sm}$ data of Ref. [5].

Table 1. Relative intensities to ${ }^{150} \mathrm{Sm}$

\begin{tabular}{|c|c|c|c|}
\hline Level & $\begin{array}{c}\mathrm{E}_{\gamma} \\
(\mathrm{keV})\end{array}$ & $\begin{array}{c}\gamma \text {-ray } \\
\text { data }\end{array}$ & $\begin{array}{c}\text { Fit to } \\
\text { t-o-f }\end{array}$ \\
\hline $0_{1}{ }^{+}$ & 0 & -- & $271(19)$ \\
\hline $2_{1}{ }^{+}$ & 334 & $100(5)$ & 100 \\
\hline $4_{1}{ }^{+}$ & 440 & $34(4)$ & $22(6)$ \\
\hline $6_{1}{ }^{+}$ & 506 & $13(2)$ & -- \\
\hline
\end{tabular}

The population strengths of the ${ }^{100} \mathrm{Ru} 0^{+}$states are being extracted and corrected for the feeding from any higher excited states. In comparison with $(\mathrm{p}, \mathrm{t})$ data [9] the first excited $\mathrm{O}_{2}{ }^{+}$state is not populated as strongly, indicating a larger $2 \mathrm{n}$ occupancy.

\section{Conclusions}

The challenge in achieving high resolution measurements using $\left({ }^{3} \mathrm{He}, \mathrm{n}\right)$ reactions is addressed by combining a $\gamma$-ray spectrometer together with a neutron detector array placed at small angles. Test experiments were performed on various systems. States with $L=0$ momentum transfer were populated very selectively and excellent signal to background ratio was achieved. This type of measurement is equivalent to the $(\mathrm{p}, \mathrm{t})$ or $(\mathrm{t}, \mathrm{p})$ reactions studied extensively with magnetic spectrometers.

These methods are complimentary and population strengths are being extracted in order to determine the $2 p$ and $2 \mathrm{n}$ occupancy of the relevant states.

\section{Future Prospects}

These initial experiments show excellent promise in providing extra experimental evidence into the structure of excited $0^{+}$states. It is surprising to see the yrast states and other states with non-zero spins so clearly. The neutron detectors are strongly selecting $L=0$ states and the only way the yrast $2^{+}, 4^{+}$and $6^{+}$states should be populated is by $L=2,4$ and 6 transitions respectively. The experimental data on ${ }^{150} \mathrm{Sm}$ suggest that the incoming ${ }^{3} \mathrm{He}$ must Coulomb or inelastically excite the target nucleus to the yrast $2^{+}, 4^{+}$and $6^{+}$states in ${ }^{148} \mathrm{Nd}$ and then subsequently have $L=0$ two proton transfer to the yrast $2^{+}, 4^{+}$and $6^{+}$states in ${ }^{150} \mathrm{Sm}$. Inelastic excitations in direct reactions have been studied extensively by Asciuitto and colleagues [11-13] but have otherwise been largely ignored.

\section{Acknowledgements}

We wish to thank the accelerator group of iThemba LABS for delivery of excellent ${ }^{3} \mathrm{He}$ beams.

This work was financially supported by the National Research Foundation of South Africa.

\section{References}

[1] Kris Heyde and John L. Wood, Rev. Mod. Phys. 83, 1467 (2011)

[2] W. D. Kulp et al., Phys. Rev. C77, 061301(R) (2008)

[3] J. F. Sharpey-Schafer et al., Eur. Phys. J. A47, 5

(2011)

[4] J. F. Sharpey-Schafer et al., Eur. Phys. J. A47, 6 (2011)

[5] W. P. Alford et al., Nucl. Phys. A321, 45 (1979)

[6] M. J. Hornish et al., Phys. Rev. C74, 044314 (2006).

[7] A. S. Barabash et al., Phys. Rev. C79, 045501 (2009).

[8] Frank T. Avignone III, Steven R. Elliott and Jonathan Engle, Rev. Mod. Phys. 80, 481 (2008).

[9] J. S. Thomas et al., Phys. Rev. C 86, 047304 (2012)

[10] S. P. Bvumbi et al., Phys. Rev. C81, 044333 (2013)

[11] R. J. Ascuitto and Norman K. Glendenning, Phys. Rev. C2, 1260 (1970)

[12] R. J. Ascuitto and Bent Sørensen, Nucl. Phys. A190, 297 (1972)

[13] R. J. Ascuitto, C. H. King and L. J. McVay, Phys. Rev. Lett. 29, 1106 (1972) 